\title{
COMMUNICATION
}

\section{Multi-site incorporation of bioactive matrices into MEMS-based neural probes}

\author{
Justin C Williams ${ }^{1,2,3}$, Matthew M Holecko II $^{1,3}$, Stephen P Massia ${ }^{1}$, \\ Patrick Rousche $^{1,4}$ and Daryl R Kipke ${ }^{1,2,5}$ \\ ${ }^{1}$ Harrington Department of Bioengineering, Arizona State University, Tempe, AZ 85287, USA \\ ${ }^{2}$ Department of Biomedical Engineering, University of Michigan, Ann Arbor, MI 48109, USA \\ E-mail: j.williams@neurosurg.wisc.edu
}

Received 18 October 2005

Accepted for publication 1 November 2005

Published 30 November 2005

Online at stacks.iop.org/JNE/2/L23

\begin{abstract}
Methods are presented to incorporate polymer-based bioactive matrices into micro-fabricated implantable microelectrode arrays. Using simple techniques, hydrogels infused with bioactive molecules are deposited within wells in the substrate of the device. This method allows local drug delivery without increasing the footprint of the device. In addition, each well can be loaded individually, allowing spatial and temporal control over diffusion gradients in the microenvironment of the implanted neural interface probe. In vivo testing verified the following: diffusion of the bioactive molecules, integration of the bioactive molecules with the intended neural target and concurrent extracellular recording using nearby electrodes. These results support the feasibility of using polymer gels to deliver bioactive molecules to the region close to microelectrode shanks. This technique for microdrug delivery may serve as a means to intervene with the initial phases of the neuroinflammatory tissue response to permanently implanted microelectrode arrays.
\end{abstract}

(Some figures in this article are in colour only in the electronic version)

\section{Introduction}

Local drug delivery to a particular area within the body is a widely held goal in the medical and biomedical engineering communities. Several advantages arise from targeting specific organs, tissues or micro-scale features at the desired site of pharmacological action. Dosage can often be drastically reduced, eliminating undue bodily stress and side effects associated with systemic delivery. By physically limiting the site of action, substances which affect multiple biological

3 Current address: Department of Biomedical Engineering, University of Wisconsin, Madison, WI 53706, USA.

4 Current address: Department of Bioeengineering, University of Illinois at Chicago, Chicago, IL 60607, USA.

5 Current address: Department of Biomedical Engineering, University of Michigan, Ann Arbor, MI 48109, USA. systems can be applied to specific purposes. This study is particularly interested in local microscale neural drug delivery.

Neural systems research stands to benefit greatly from targeted drug delivery. The blood-brain barrier presents a challenge to systemically administered substances, often increasing the necessary dosage. In addition, neural tissues of similar cellular composition often have drastically different functions based on anatomical location. This study focuses on chronically implanted electrically-based neural interface probes. These devices often suffer a loss of function over a period of weeks to months because the neuroinflammatory response produces a glial sheath surrounding the implant, electrically isolating the probe from the surrounding tissue [1]. Local drug delivery to the region surrounding the implant as a means to pharmacologically intervene with this process is being actively pursued by several groups [2-5]. 
In general, local microscale drug delivery to the tissue surrounding neural implants has followed two basic approaches. One method involves fabricating microfluidic channels into the substrate of the probe allowing injection of drugs into the tissue immediately surrounding the opening [2, 5]. The other technique relies on polymer surface coatings which support timed release of bioactive chemicals [4, 6, 7]. Both are successful, but have significant drawbacks. Integrated microfluidic channels greatly complicate probe production, leading to lower yields and more expensive fabrication costs. In addition, the channels increase the footprint, or size, of the device, which may further injure the surrounding tissue during the implantation procedure. Polymer surface coatings could increase the device's dimensions as well, potentially adding a layer to the surface that could range from tens to hundreds of micrometers in thickness. The high surface area-to-volume ratio of the coatings may reduce the ability for sustained delivery over long periods of time, which could minimize its potential for limiting the chronic neuroinflammatory response.

We present a method for targeted microscale drug delivery, based on the polymer coating method, which eliminates some of the disadvantages associated with conventional techniques. In this method, micromachined 'wells' are fabricated into the substrate of a MEMS (micro-electro-mechanical systems)based chronic implant. These 'wells' are holes that extend through the device's thickness and allow for the integration of matrices (i.e. hydrogels) infused with bioactive substances. Since the matrices replace substrate instead of adding to it, the device's footprint is not increased. The lower surface area-to-volume ratio lends the technique to extended timerelease periods. Most significantly, the release sites are localized within micrometers of individual electrodes, and each well can be loaded with a different material, leading to spatial and temporal control over diffusion profiles through the surrounding microenvironment.

Numerous side-benefits arise from the technique as well. Since the electrode is within microns of the well, gradients of growth factors could be established to direct neurite growth toward the recording site. The design of the wells permits tissue growth through the substrate, which may anchor the probe more firmly in place. Finally, this method of local drug delivery extends beyond chronic neural implants. The technique's simplicity and effectiveness make it easily adaptable to a wide range of implantable microdevices.

In summation, this research seeks to explore the feasibility, consistency and functionality of this novel method for targeted microscale drug delivery. First, this technique's feasibility will be validated by adapting manufacturing processes so that devices are constructed with the aforementioned wells and then developing methods with which these wells can be filled with various bioactive matrices. Next, we will investigate the consistency of these methods by determining how reliably these devices can be fabricated and how consistently the wells can be filled with a bioactive matrix. Lastly, we will test the functionality of these devices by using in vivo experiments to determine if the matrix is delivered to the desired tissue, whether the devices survive implantation and if the devices remain electrically viable. By answering these questions, we will provide a solid foundation for future use of these techniques in experiments attempting to minimize the inflammatory response to the implantation of neural prosthetic devices.

\section{Materials and methods}

Fabrication of the silicon- and polymer-based probes used in this study has been previously described [8, 9]. Both types of devices are planar structures designed to include small wells of various shapes and sizes in the probe's substrate. These wells are typically located within 30 micrometers of a recording site, although the exact placement is a variable design parameter. The wells are rectangular or elliptical with dimensions ranging from 20 to $60 \mu \mathrm{m}$ wide and extending the depth of the substrate, generally $7 \mu \mathrm{m}$ to $20 \mu \mathrm{m}$ deep.

\subsection{Gels}

To demonstrate the robustness of the technique, three types of matrices were used in order to cover a range of material properties, including viscosity and release rates. The gels utilized in this study were NeuroTrace ${ }^{\circledR}$ Tissue Labeling Paste (Molecular Probes Inc., Eugene, OR), NeuroSeal dural sealant (NeuroNexus Technologies, Ann Arbor, MI) and polypropylene glycol. In addition to using unmodified gels, other substances were added to the matrices to demonstrate the delivery and release of bioactive chemicals. To this end, NeuroTrace ${ }^{\circledR}$ pastes containing lipophilic fluorescent tracers DiI, DiO and DiD (stock from Molecular Probes, Inc.) were used. Lyophilized nerve growth factor (NGF, Sigma Inc.) was added to NeuroTrace ${ }^{\circledR}$ paste that did not contain fluorescent dye. Green dye was mixed into unreacted alginate portion of the NeuroSeal and polypropylene glycol to provide for visualization of the normally translucent materials. Both alginate and polypropylene glycol were applied to the probes in their liquid states.

\subsection{Method to fill probe wells}

Two basic micropipetting methods were developed to selectively and reliably fill individual probe wells with the gels (figure 1(A) and (B)). For both, micropipettes of various tip sizes and lengths were fabricated from borosilicate glass tubing using a micropipette puller (Sutter Instruments, Navato, CA). The typical inside tip diameter was approximately $10 \mu \mathrm{m}$, although this was adjusted according to material viscosity and well dimensions. The 'injection method' for filling wells with the polypropylene gel involved aspirating the liquid into a micropipette and then injecting it, under pressure, into the wells (figure 1(A)). The second method, 'droplet deposition' (figure 1(B)), involved suspending a drop of gel from the micropipette tip and then depositing the drop into the well by bringing the gel into contact with the probe using micropositioners (Eppendorf, Westbury, NY). The droplet deposition method was the preferred approach for the more viscous NeuroTrace ${ }^{\circledR}$ and NeuroSeal gels. In both methods, 


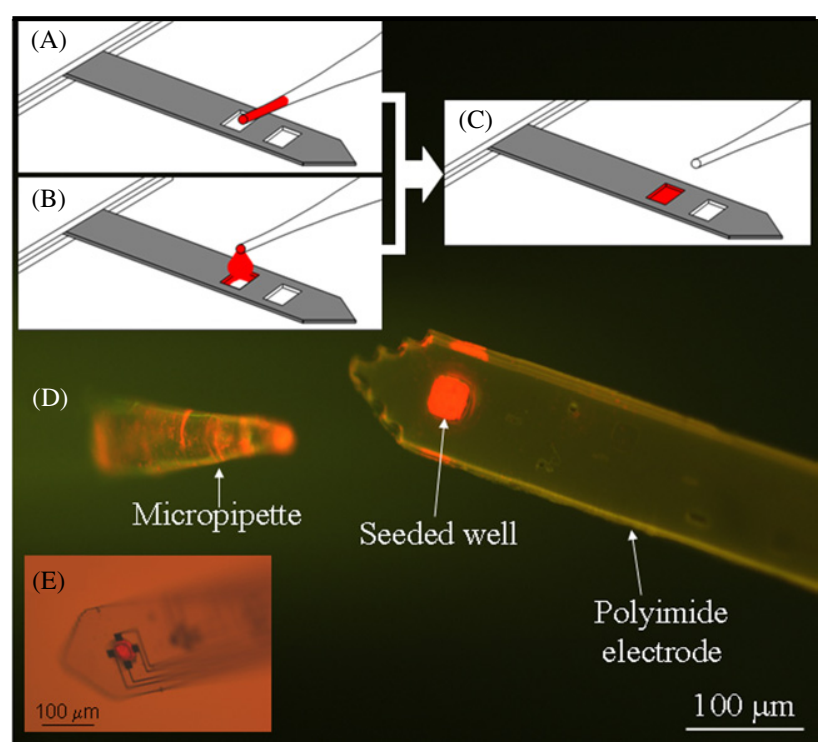

Figure 1. Illustration of electrode well seeding technique. Schematics of the device and pipette depicting the injection and droplet deposition methods prior to gel loading are given in (A) and (B), respectively, (C) schematic of a device with a filled well, (D) fluorescent image of probe immediately after the gel loading and subsequent withdrawal of pipette tip. The edges of the probe appear red due to internal reflection of the fluorescing DiI emanating from the well through the polyimide substrate. The volume of gel contained within the well is approximately 9 picoliters based on the well dimensions and (E) shows a polyimide based electrode with a seeded well in the middle of a tetrode arrangement of recording sites.

the probe shank was suspended by clamping it between microscope-slide-cover slips to prevent capillary forces from pulling fluid from the well along the probe's bottom.

All animal-use procedures were approved by the Institutional Animal Care and Use Committee at Arizona State University.

\section{Results}

Two techniques for filling micromachined wells within MEMS devices, injection and droplet deposition, were evaluated. With proficiency, both were simple to perform, taking approximately 30 minutes for set-up, filling four wells with a single-gel type and probe removal. Both techniques produced high yield, with proper filling of four wells on a multishank probe occurring greater than $90 \%$ of the time. Precise volume control was not necessary, as both injection and droplet deposition filled the wells through capillary action. Loading separate gels into different wells was easily achieved (figure 2(A) and (B)), as was loading two gels into a single well (figure 2(C) and (D)). However, increasing the number of gel types decreased yield and increased filling time per device.

Four gel types were successfully loaded into wells in both silicon and polyimide devices. To deliver high viscosity gels, droplet deposition was the preferred technique since aspiration into and ejection from a small pipette tip using high pressure was difficult and inaccurate. For lower viscosity gels, either injection or droplet deposition could be used. In
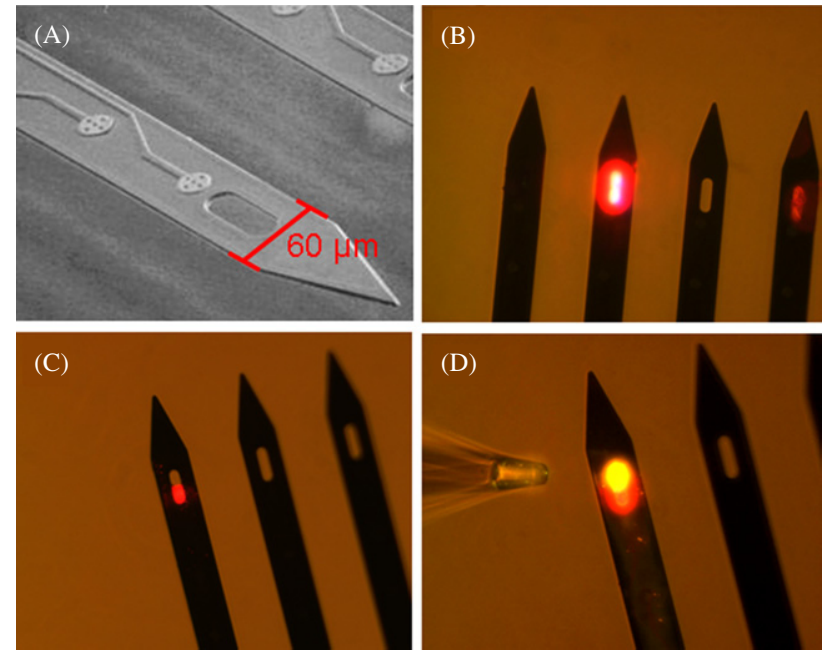

Figure 2. Seeding of multi-well devices with different gels. (A) SEM micrograph of the base microelectrode technology. All devices are $60 \mu \mathrm{m}$ wide as shown, (B) loading of multiple wells, each with a different gel, from left to right: polypropylene glycol (green hue not visible due to microscope and camera settings), DiI gel (bright red), no gel and calcium alginate (translucent red),

(C) focal delivery of DiI gel into one half of a silicon probe well and (D) fluorescent image of a device immediately after focal delivery of $\mathrm{DiO}$ gel into remaining half of the well. DiI fluorescence is shown in red and $\mathrm{DiO}$ is shown in orange (in online version only).

both cases, the tip size of the pipette needed adjusting based on gel properties (primarily viscosity) to deliver the proper volume to the well. For the types and sizes of probes used, neither well geometry nor probe substrate material influenced the loading techniques. It was outside the aim of this study to exhaustively explore all well geometries, gel types and probe configurations. Evaporation during the process was both a hindrance and a benefit. When occurring in the droplet at the pipette tip, it concentrated the gel and occasionally clogged the pipette. However, once deposited within a well, evaporation solidified the gel in the proper location.

For proof-of-concept, several in vivo tests were carried out on only a small number of animals $(n=2-5)$ to evaluate the reliability of the device insertion and determine the feasibility of using them in future, more thorough experiments. For these experiments, seeded devices were UV-sterilized and implanted in the somatosensory or motor cortex of anesthetized rats using previously described surgical techniques [9]. At the end of the evaluation period, each animal was euthanized and its brain tissue prepared for subsequent histological analysis using previously published methods in a complementary study $[1,10]$. Histological sections were imaged using transmitted light, fluorescent and confocal microscopy techniques to verify the techniques only. While no quantitative assessments were carried out, qualitative evaluations were made on a few samples. Additionally in some instances, brief daily neural recordings were obtained for post-implantation periods of up to four weeks using conventional neural recording procedures for extracellular field potentials and spiketrains $[8,9]$. These recordings were taken only to verify electrode functionality after matrix seeding and implantation, but were not quantitatively analyzed for signal characteristics. 


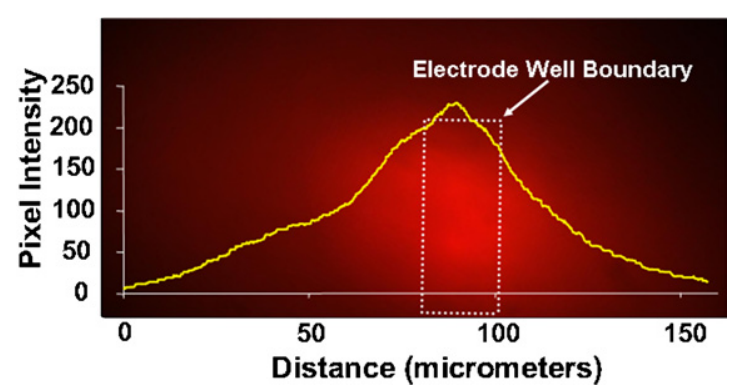

Figure 3. Delivery of gel-seeded device into neural tissue. The background of this figure shows an image taken using fluorescent illumination where diffusion of the DiI gel from an implant can be seen four hours post implantation. The dashed white rectangle represents the approximate position of the electrode well in the tissue. The plot (overlaid in yellow) shows the intensity profile for diffusion of the DiI measured from the center of the well along the horizontal axis.
As seen in figure 3, gels remained inside the wells throughout the implantation process. For a low percentage of the time, some of the gel became dislodged during implantation and was spread along the probe's surface. Once in place, the material within the wells began diffusing into the tissue surrounding the electrode. A time-dependent diffusion profile, controlled by the properties of the gel and solute, was thus achieved (figure 3). This diffusion profile (for DiI only) was determined by measuring the intensity profile for the fluorescence intensity of the DiI from the center of the well along its horizontal axis. Actual concentrations were not determined as what appears to be a homogenous mixture on the macroscale is often not when viewed on the microscale. To demonstrate localized delivery of a bioactive substance, figure 4 shows the results of an implant in which the well in one shank of a four-shank probe was filled with NeuroTrace ${ }^{\circledR}$ mixed with lyophilized NGF. Following a 40-day post-implant
(A)
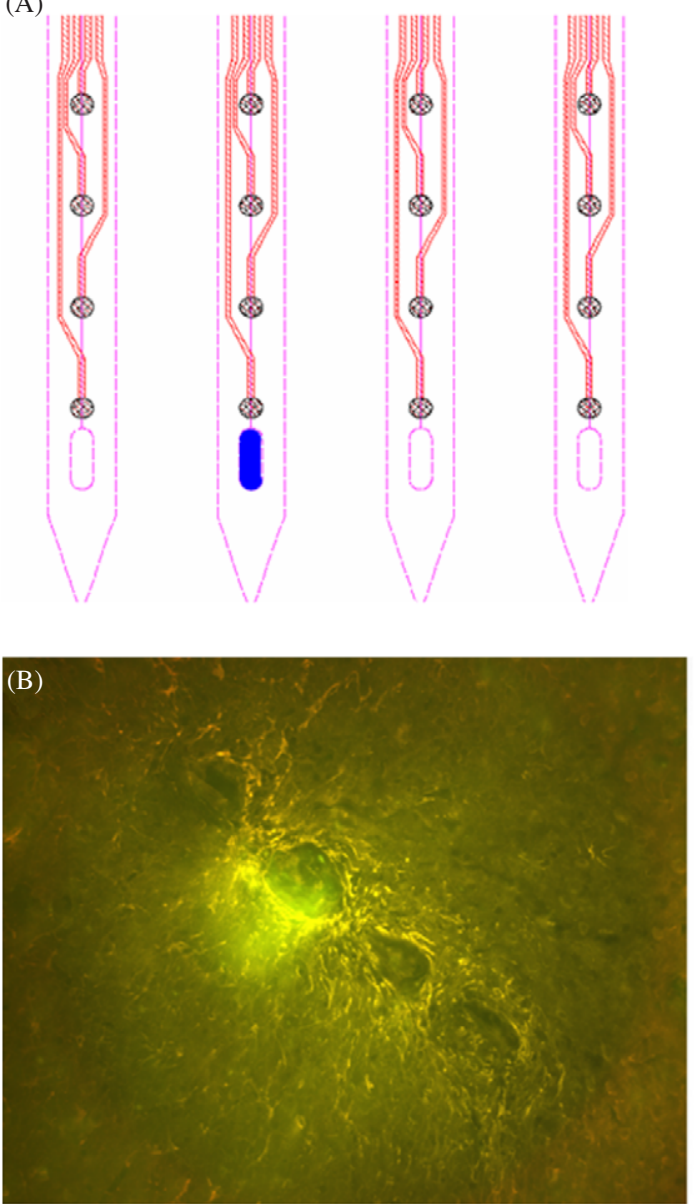

(C)
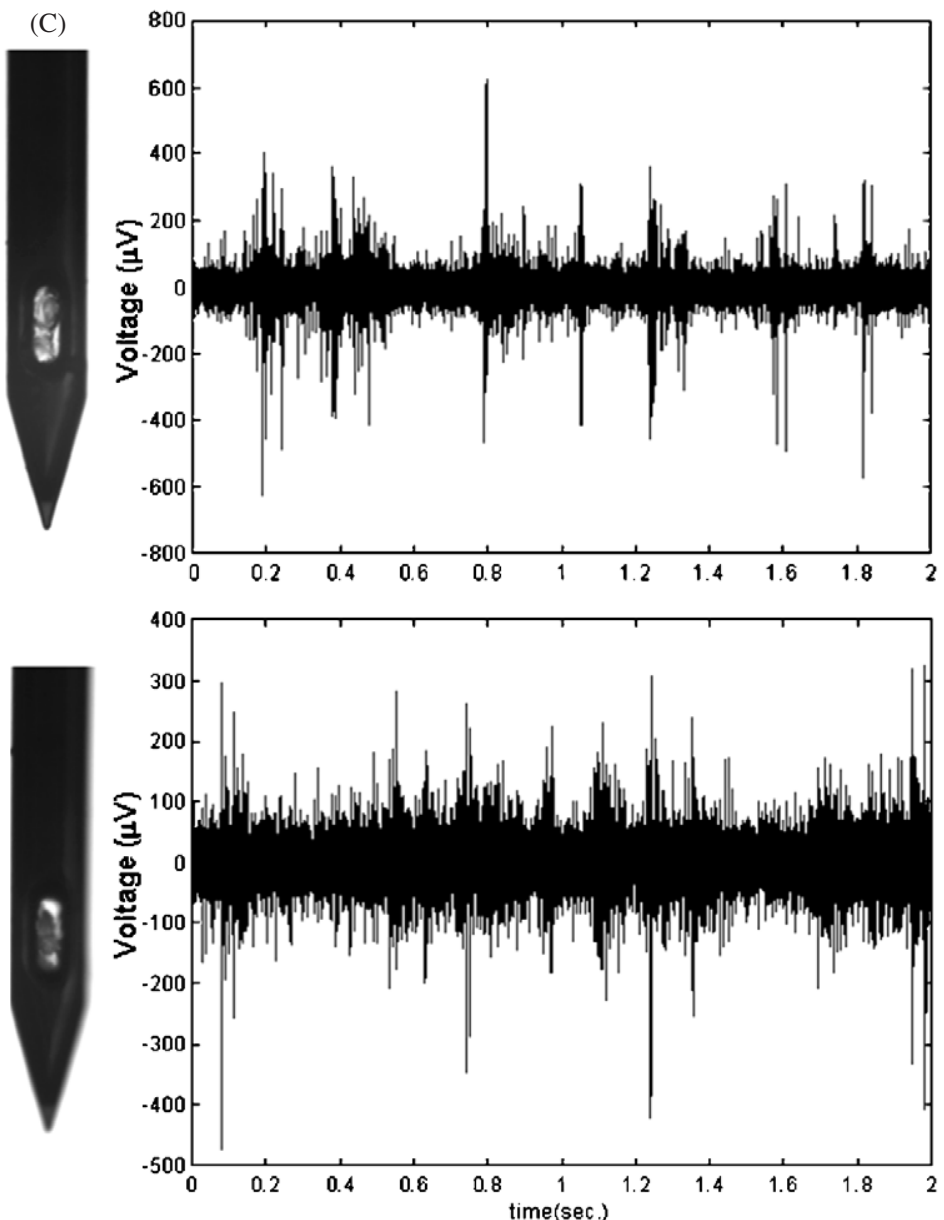

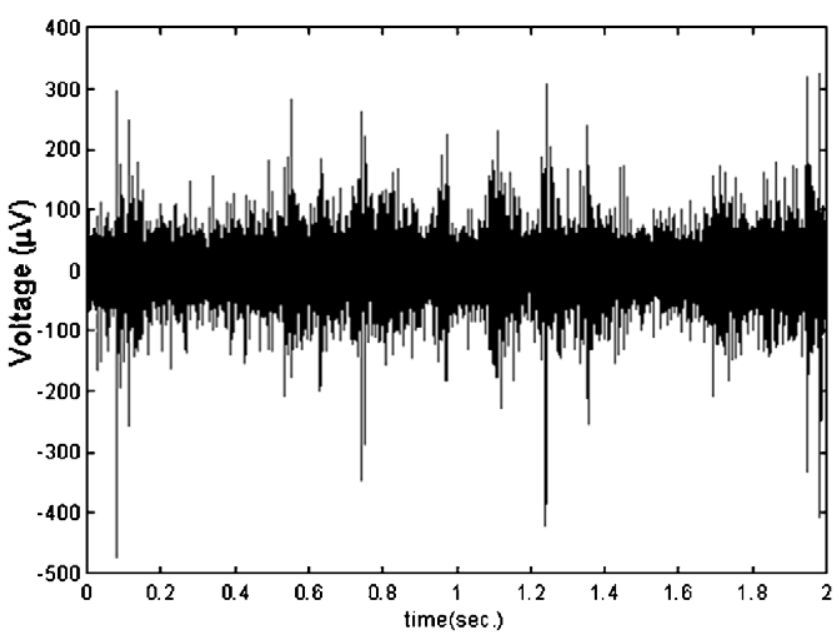

Figure 4. Wells seeded with NGF. The upper left schematic (A) illustrates the layout of an implanted microelectrode array, showing the relative position of the NGF-seeded well in relation to a recording site. In this four-shank implant, only one of the shanks has an NGF-seeded well. The implanted animal was sacrificed after 40 days, sectioned at the level of the NGF-seeded well, and immunohistochemically stained with antibodies against GFAP and the extracellular matrix protein, laminin. The lower left figure in this layout (B) is a histological image from this implant. The site that contained the NGF-seeded well (second from the left in this image) showed increased levels of matrix deposition versus the unseeded electrode shank sites. Part (C) shows the electrophysiological recordings taken from two individual electrode sites closest to two different seeded wells, each at 40-days post-implantation. The inset image to the left of each recording shows the pre-implant electrode with lyophilized NGF seeded in each well. 
period, the animal was euthanized for histological analysis. Qualitative observations of figure 4(B) show a notable tissue response in the form of laminin deposition seen as a green 'cloud' over and adjacent to the site of NGF release from the gel. Additionally, an increased level of GFAP expression (yellow stained cells) can be seen around the implant sites. These results are typical for the sites of NGF seeding $(n=4)$, but noticeably different from the well sites without NGF $(n=28)$, which all showed a relatively normal response (in comparison to previous studies [8]). A two-second sample of the extracellular recordings taken from two different recording sites, each located 15 micrometers from two different seeded wells is given in figure $4(\mathrm{C})$. These recordings demonstrate that the sites near the NGF-seeded wells remain electrically viable following implantation. The recordings shown are typical for the sites adjacent to the NGF-seeded wells, although not statistically different from the other sites in the array. In all the animals that were implanted with functioning electrodes ( $n=4,48$ hours and 40 days), neural signals were recorded from the sites adjacent to the wells that had been seeded with bioactive molecules. Qualitatively, the electrodes were able to record unit activity with acceptable signal amplitude (figure 4), as would be expected from the investigators' experiences with these types of electrodes [8].

\section{Discussion}

This study describes a new technique for multi-site, localized drug delivery using penetrating neural probes. First, wells are micromachined into the device substrate at various sites. Next, a hydrogel containing a bioactive substance is loaded into each well individually. Upon implantation, the hydrogel releases the drug via diffusion into the surrounding tissue. The combination of these features results in several advantages over conventional drug-delivery techniques such as polymer coatings or microfluidic channels for fluid injection.

The use of micromachined wells provides a number of benefits. Perhaps the most obvious advantage is the ability to integrate bioactive substances into the device without increasing the footprint, or cross-sectional area, of the implant. Both surface coatings and integrated microfluidic channels increase the device dimensions, potentially leading to increased tissue damage during implantation and a larger volume of foreign materials remaining lodged within the tissue. In contrast, wells located in the probe substrate do not increase tissue damage during the implant procedure, and a smaller volume of foreign material is left within the tissue once the hydrogel has dissipated. Another benefit arises from the possibility of inducing cellular growth through the holes, which, if achievable, may serve to physically anchor the implant into the surrounding tissue. Particularly for electrically-based probes, any reduction of device movement post-implantation would aid in the localization of electrical signals and their subsequent assignment to individual cells.

Another important aspect of this method is the general ease and success of the well-filling techniques. Since the methods are effective regardless of substrate material, well geometry and gel/drug material, these variables remain unconstrained as design parameters. For example, consider time-controlled drug release into the local tissue surrounding the implant. Compared to polymer surface coatings, hydrogels deposited in wells have a much lower surface area-to-volume ratio, leading to a more prolonged diffusion profile. Prolonged release is often desirable when attempting to control conditions such as the neuroinflammatory response over a span of hours to days.

However, the true benefit regarding time-controlled drug release is realized through the ability to potentially manipulate the diffusion profile through variable parameters such as well geometry and gel material. Hydrogels contained within small aspect-ratio holes have low surface area-to-volume ratios, leading to slower drug release rates. A larger aspect-ratio hole would increase the surface area-to-volume ratio, thus speeding up diffusion into the tissue [11, 12]. Additionally, gels with differing properties may be used to influence both the duration and rate of drug release.

The ability to precisely orient micromachined wells in implantable devices opens up a wide variety of experimental possibilities. Because the wells are formed as an integral part of the microelectrode fabrication process, the position and geometry of each well is a changeable design parameter. Each well, depending on geometry, can deliver volumes (in the range of tens of picoliters) to a location known with micrometer accuracy. The amount of affected tissue surrounding this location can be controlled by adjusting the volume, concentration and release profile of the gel to coincide with the time course of the biological events. As illustrated in this study, chemical gradients (figure 3) can be centered within micrometers of electrode sites (figure 1(D)). Therefore, it may be feasible to attract neural growth to the specific location of an electrode using growth factors and other chemical signaling cues. Recording site proximity is particularly important if the goal of the implant procedure is to measure the effect (e.g. neurite extension, neural modulation, etc.) of the bioactive molecule on neurons [5, 13, 14].

It was not a forgone conclusion that delivery of bioactive molecules would not interfere with the adjacent electrodes' ability to record unit activity. As seen in figure 4, the electrodes remain electrically viable and continue to record neural activity as the bioactive molecules diffuse into the local extracellular space. In all of the animals that were implanted with electrically functional implants $(n=4)$, the electrodes adjacent to the seeded wells recorded neural activity (figure 4 is a typical response). This verifies that the process of filling the wells and the subsequent diffusion of molecules into the extracellular space does not appear to preclude the electrodes' ability to record extracellular neural activity. The long-term effects of diffusion of the bioactive molecules are yet to be determined and will be the focus of future studies.

The capability for localized drug delivery is extended by the use of multiple wells. By nature of the well-filling technique, each well is loaded with polymer individually. Utilizing wells containing various bioactive material formulations allows the creation of a spatially and temporally distributed chemical profile in the microenvironment of the device. In this manner, a probe could deliver different drugs 
from selected sites, each with an independent delivery rate profile [15].

The studies presented here hint at this technique's potential for use in delivering substances to neural tissue in order to reduce inflammation, stimulate or minimize cellular responses or treat neurological disorders. However, this technique has already been practically implemented in a concurrent study where these methods are used to deliver fluorophores to neural tissue for visualization purposes. (See 'Visualization of the intact interface between neural tissues and implanted microelectrode arrays' in this issue [10].) This example illustrates the usefulness of this technique and demonstrates its potential in secondary applications such as in vivo imaging, anatomical tracing and electrode position validation.

\section{Conclusions}

This study presents a straightforward method to achieve local, time-controlled, multi-site microscale drug delivery within a single device. Micromachined wells provide precise spatial control, decrease the volume of the implant and, unlike other techniques, do not require an increased footprint to achieve delivery of bioactive substances. The simplicity of the wellfilling technique makes it amenable to multiple types of probe materials and drug-infused polymers. Filling multiple wells on a single probe with different substances allows greater control of spatial and temporal drug release than is otherwise possible. The major benefit of the work is to extend the design parameters of chronic implantable neural interface devices, giving the researcher greater control over multiple aspects of probe functionality.

\section{Acknowledgments}

The authors acknowledge the contributions of David Pellinen, David Andersen, Jamille Hetke, Tim Becker and Rio Vetter at the University of Michigan, William Shain at the Wadsworth Center in Albany, NY; Tedd Brandon, Diane LoBaido, Gholam Ehteshami, Dennis McDaniel, and the W M Keck Bioimaging Laboratory at Arizona State University and Thomas Pearce at the University of Wisconsin-Madison. Primary funding was provided by the DARPA-Bio/Info/Micro Program.
Additional funding was provided by the University of Wisconsin-Madison College of Engineering and Graduate School.

\section{References}

[1] Turner J N et al 1999 Cerebral astrocyte response to micromachined silicon implants Exp. Neurol. 156 33-49

[2] Retterer S T et al 2004 Model neural prostheses with integrated microfluidics: a potential intervention strategy for controlling reactive cell and tissue responses $I E E E$ Trans. Biomed. Eng. 51 2063-73

[3] Shain W et al 2003 Controlling cellular reactive responses around neural prosthetic devices using peripheral and local intervention strategies IEEE Trans. Neural Syst. Rehabil. Eng. 11 186-88

[4] Cui X Y et al 2003 In vivo studies of polypyrrole/peptide coated neural probes Biomaterials 24 777-87

[5] Chen J et al 1997 A multichannel neural probe for selective chemical delivery at the cellular level IEEE Trans. Biomed. Eng. 44 760-69

[6] He W and Bellamkonda R V 2005 Nanoscale neuro-integrative coatings for neural implants Biomaterials 26 2983-90

[7] Cui X Y et al 2001 Surface modification of neural recording electrodes with conducting polymer/biomolecule blends J. Biomed. Mater. Res. 56 261-72

[8] Vetter R J et al 2004 Chronic neural recording using silicon-substrate microelectrode arrays implanted in cerebral cortex IEEE Trans. Biomed. Eng. 51 896-904

[9] Rousche P J et al 2001 Flexible polyimide-based intracortical electrode arrays with bioactive capability IEEE Trans. Biomed. Eng. 48 361-71

[10] Holecko II M M, Williams J C and Massia S P 2005 Visualization of the intact interface between neural tissue and implanted microelectrode arrays J. Neural Eng. 2 97-102

[11] Agrawal C M and Ray R B 2001 Biodegradable polymeric scaffolds for musculoskeletal tissue engineering J. Biomed. Mater. Res. 55 141-50

[12] Masri B A et al 1995 Effect of varying surface patterns on antibiotic elution from antibiotic-loaded bone-cement J. Arthroplasty 10 453-59

[13] Kennedy P R 1989 The cone electrode: a long-term electrode that records from neurites grown onto its recording surface J. Neurosci. Methods 29 181-93

[14] Kennedy P R, Bakay R A and Sharpe S M 1992 Behavioral correlates of action potentials recorded chronically inside the cone electrode Neuroreport 3 605-8

[15] Kikkinides E S et al 1998 A two-phase model for controlled drug release from biphasic polymer hydrogels J. Control. Release 51 313-25 